ISSN: $1980-055 X$

\title{
UTILIZAÇÃO DE SIG NA ANÁLISE DE TORNADOS: UMA FER- RAMENTA METODOLÓGICA PARA O BRASIL
}

\author{
Isabela Pena Viana de Oliveira Marcelino e Lucí Hidalgo Nunes²
}

\begin{abstract}
RESUMO
As ocorrências de tornados e de outros eventos atmosféricos extremos sempre aconteceram no território brasileiro. Entretanto, somente nos últimos anos têm advindo maiores enfoques e pesquisas relacionadas a esse fenômeno no país. Ainda não foi possível afirmar se o incremento na freqüência e intensidade de tornados está vinculado a maior divulgação dada pelos meios de comunicação ou as mudanças globais. Essa incógnita existe em decorrência da ausência de um banco de dados para as ocorrências de eventos extremos no Brasil. Uma das ferramentas que tem sido grandemente utilizada para análise de fenômenos que causam desastres é o Sistema de Informação Geográfica (SIG). Essa ferramenta é extremamente eficiente tanto na fase de alerta, quanto na fase de análise e gerenciamento de risco. Ressalta-se que o SIG também pode ser utilizado para análises atmosféricas a priori (origem) e análises de superfície a posteriori (conseqüências). Neste contexto, o presente trabalho tem como objetivo verificar a aplicabilidade do SIG como ferramenta para análise de episódios de tornados no Brasil. Destaca-se que existe uma grande dificuldade na padronização de um banco de dados de tornados para o país, em função da adoção de variáveis que sejam representativas e confiáveis. São estas variáveis que permitirão o melhor entendimento da origem e das conseqüências desse fenômeno.
\end{abstract}

Palavras chaves: tornado, SIG, banco de dados, Brasil.

\section{USE OF GIS IN TORNADO ANALYSIS: A METHODOLOGICAL TOOL FOR BRAZIL}

\begin{abstract}
Tornadoes and other extreme atmospheric events have always occurred in the Brazilian territory. However, researches related to those phenomena in the country have appeared only in the last years. It is not possible to affirm that increase of the frequency and intensity is linked to the more information by the media, or associated to global change. These uncertainties exist due to the lack of a standard database for the occurrences of extreme events in Brazil. Geographical Information System (GIS) is one of the tools that has
\end{abstract}

${ }^{1}$ Geógrafa, Doutoranda em Geografia, Instituto de Geociências - UNICAMP, CP 6152 CEP 13083970, isabelam@ige.unicamp.br.

${ }^{2}$ Geógrafa, Professora Doutora Titular do Departamento de Geografia, Instituto de Geociências UNICAMP, CP 6152 CEP 13083-970, luci@ige.unicamp.br. 
been largely used for disaster events. It is extremely efficient to both the alert phase and the analysis and risk management. It is emphasized that GIS can also be used to atmospheric analyses a priori (origin) and surface analyses a posteriori (consequences). In this context, the present work aims to verify the applicability of GIS for analysis of tornado episodes in Brazil. Currently, there is a big difficulty to create a standard and reliable tornadoes' database in the country. Thus, it is necessary to establish representative variables for a comprehensive understanding of the origin and consequences of this kind of phenomenon.

Key words: tornado, GIS, database, Brazil.

\section{Introdução}

Os eventos atmosféricos extremos são os grandes deflagradores de desastres naturais no território brasileiro. Por ocorrerem de forma súbita e, em sua maioria, não apresentarem um padrão cíclico e facilmente reconhecível, acabam dificultando a realização de prognósticos confiáveis. Dentre esses fenômenos, os tornados têm se destacado pela sua velocidade e violência, causando grandes perdas socioeconômicas nas áreas afetadas.

O tornado é definido como uma intensa coluna de ar giratória pendente de uma nuvem cumuliforme até a superfície terrestre, sendo normalmente visível na forma de uma nuvem funil (GLICKMAN, 2000). São originados de instabilidades intensas, formados por um ambiente em escala sinótica que se caracteriza por movimentos verticais ascendentes, elevadas temperatura e umidade atmosférica (DOSWELL e BOSART, 2000). Conhecer os padrões atmosféricos propícios para a formação de tornados é um dos primeiros passos para auxiliar na previsão de tempo severo (NASCIMENTO, 2005). Aliado a isso, o reconhecimento das áreas mais favoráveis à ocorrência desses fenômenos, contribuirá significativamente para o estabelecimento de medidas preventivas que possam diminuir a vulnerabilidade das áreas mais impactadas.

Uma das ferramentas que apresenta um grande potencial para a solução desta problemática é o Sistema de Informação Geográfica (SIG). O SIG permite que, em um mesmo ambiente de trabalho, sejam realizadas diversas correlações entre diferentes variáveis geofísicas e socioeconômicas, em diversas escalas de análise (BURROUGH e McDONNELL, 1998). Conforme Coppock (1995), esta ferramenta já tem sido um importante instrumento para análise e prevenção de desastres naturais. O autor comenta que o SIG é extremamente útil na identificação de áreas de risco e no monitoramento e previsão de eventos naturais extremos. Ressalta-se que esses eventos não podem ser evitados, mas seus impactos podem ser minimizados por meio da adoção de medidas preventivas, por exemplo, a implantação de sistemas de alertas, elaboração de planos de evacuação, entre outras ações emergenciais.

Segundo UNDP (2004), para o desenvolvimento de uma análise de risco, alguns dados são considerados fundamentais, como: a) características do fenômeno (tipo, magnitude, duração, etc.); b) local de ocorrência (topografia, 
hidrologia, uso da terra, climatologia, etc.); e c) elementos expostos, que são potencialmente vulneráveis (população, infra-estrutura, edificações, indicadores socioeconômicos, etc.). No que se refere às ocorrências de tornados, todos esses dados podem ser facilmente obtidos e inseridos na análise de risco. Além disso, a avaliação dos danos causados pelos tornados é fundamental para entender o comportamento e classificar a intensidade dos mesmos. Verifica-se em todo esse processo a relevância da utilização do SIG nas diferentes fases que envolvem um desastre causado por um tornado, isto é, antes (prevenção e mitigação), durante (resposta) e depois (reconstrução) (Figura 1).

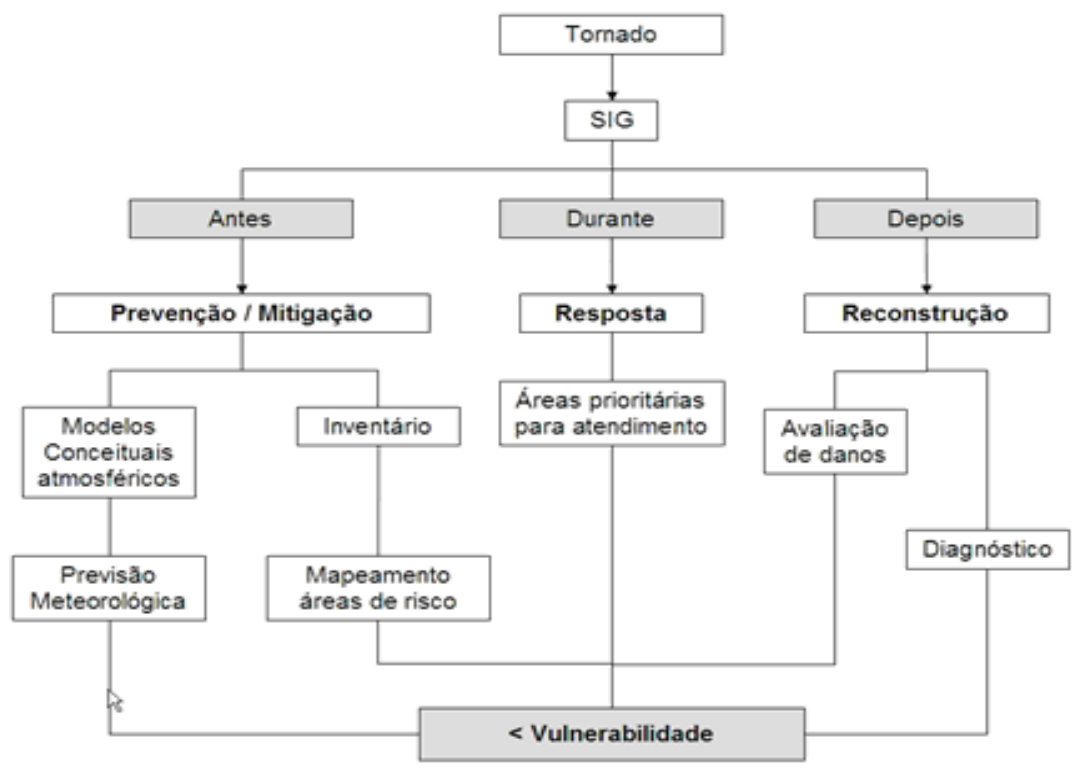

Figura 1. Esquema para mitigação dos danos e prejuízos causados por tornados em ambiente SIG.

Entretanto, a utilização do SIG na fase de resposta já tem sido amplamente difundida em algumas áreas do globo (FEMA, 2005a; b), mas na fase de diagnóstico e prognóstico atmosférico pouco do seu potencial tem sido explorado (SHIPLEY, 2005). Contudo, como citado por Coppok (1995), o campo dos desastres naturais é multidisciplinar, o que remete à necessidade de adoção de diversos tipos de dados e ferramentas de análises para melhor entender o comportamento de um dado fenômeno, e assim contribuir para a implantação de medidas eficientes nas diferentes fases de um desastre.

Neste contexto, o presente artigo tem como objetivo analisar a aplicabilidade do SIG para a análise de episódios de tornados no Brasil. Para tanto, na seção 2 será abordada a necessidade da formação de um banco de dados de tornados para o país, ressaltando as dificuldades e exemplos de bancos existentes em outras regiões do mundo. Na seção 3 será tratada a aplicabilidade propriamente dita do SIG para análise desse fenômeno, tanto na origem quanto na 
análise das conseqüências.

\section{Banco de dados de tornados no Brasil}

A formação de um banco de dados é o primeiro passo a ser dado para possibilitar a utilização do SIG nas diferentes etapas que envolvem a ocorrência de tornados. Para tanto, é imprescindível que o banco seja alimentado com dados confiáveis, compostos, preferencialmente, por longas séries históricas (SAMUEL et al., 2004). Só assim será possível compreender a dinâmica desse fenômeno, que é tão súbito, irregular e violento. Além disso, o banco de dados deve conter informações sobre as condições atmosféricas que os desencadearam, bem como informações relativas à superfície do terreno e à natureza socioeconômica das áreas impactadas.

A formação de um grande banco de dados para o Brasil ainda não foi possível, devido principalmente a ausência registros oficiais e a necessidade de uma padronização das variáveis a serem analisadas. No entanto, algumas tentativas de formação de banco de dados de tornados no país têm já foram realizadas. Nechet (2002) reuniu vários episódios de tornados em diferentes estados da nação, obtidos através de relatos observacionais, fotografias e filmagens. Foi registrado por esse autor tornados no Pará, Pernambuco, Mato Grosso do Sul, São Paulo, Paraná, Santa Catarina e Rio Grande do Sul. Oliveira (2000) e Marcelino et al. (2006) realizaram um levantamento sistemático dos episódios de tornados e trombas d'água ocorridos em Santa Catarina no período de 1976-2000. Os dados foram coletados dos relatórios de avaliação de danos (AVADAN) da defesa civil estadual, como também através de fotografias, filmagens e jornais. Esses eventos também foram classificados de acordo com a escala Fujita, com intensidades que variaram de F0 a F3.

Acredita-se que a sistematização nas análises de tempestades severas na América do Sul seja fundamental para o entendimento das instabilidades atmosféricas e, assim, contribuir com a previsão meteorológica e com o estabelecimento de medidas preventivas eficazes. Nascimento e Doswell (2005) fizeram uma analogia de como o banco de dados de tempestades dos EUA foi formado e quais são as condições atuais para a criação de um banco adequado para a América do Sul. Esses autores comentam que uma das principais ações é a formação de equipes de observação, treinadas especificamente para identificar os diferentes tipos de eventos, o que resultaria em dados confiáveis para alimentar o banco.

Por exemplo, a base de dados de tornados nos EUA possui registros desde 1916. Através da Figura 2, é possível observar que houve um significativo incremento a partir da década de 50 no século XX. Segundo Doswell et al. (1999), este aumento foi devido ao grande interesse da população e do meio acadêmico em participar de programas de treinamento para identificação e registro de tornados e fenômenos correlatos. Esses observadores treinados passaram a enviar seus dados ao SELS (Severe Local Storms Forecasting Unit) que estava vinculado ao serviço público de previsão de tempestades do United States Weather Bureau, contribuindo significativamente para a formação do banco de dados americano. 


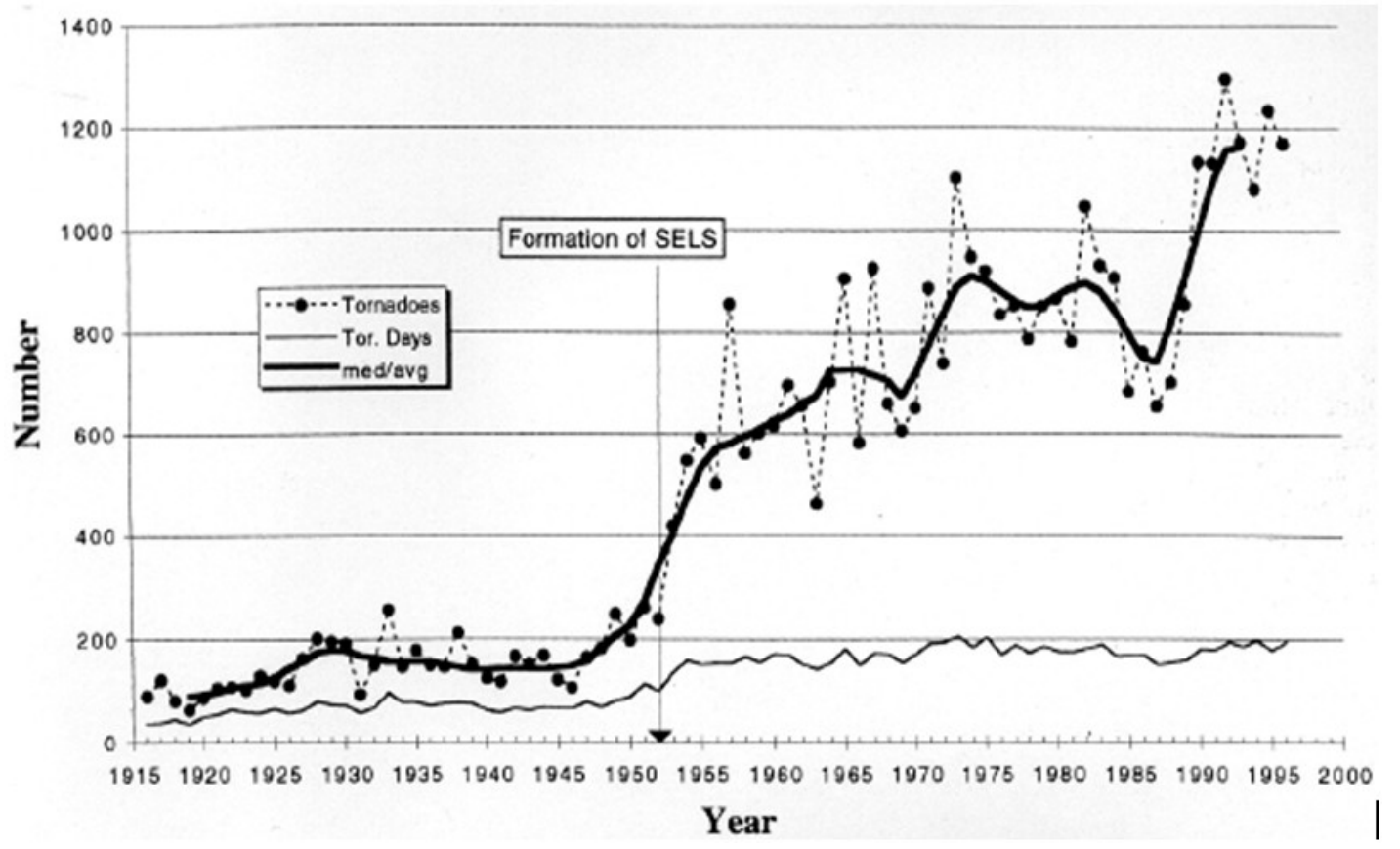

Figura 2. Registros anuais de tornados nos EUA no período de 1916 a 1995 . Fonte: Doswell et al. (1999).

É sabido que as tempestades severas nem sempre desencadeiam tornados. Assim, a formação de banco de dados de tempestades também é fundamental para que outros tipos de eventos extremos que causam desastres (vendaval, granizo, etc.), possam ser analisados e compreendidos. Como exemplo, cita-se o Storm Data, que reúne o registro oficial de vários tipos de fenômenos extremos gerados principalmente por tempestades nos EUA (NCDC, 1995). Verifica-se nesse banco de dados que apesar dos diferentes tipos de fenômenos extremos documentados, adotou-se um mesmo padrão de informação. Na Figura 3, é apresentado o registro de um tornado F0 ocorrido em Coffee County, Alabama, que destruiu um trailler (mobile home), deixando uma pessoa ferida. As informações sobre esse fenômeno vão desde localização, data, horário local, extensão e comprimento do rastro, até número de pessoas mortas e feridas, estimativa dos danos e natureza da tempestade. Abaixo dessas informações, são descritas algumas características relevantes sobre a ocorrência do fenômeno e/ou das suas conseqüências. Outros bancos de dados de tornados podem ser encontrados em outros estados americanos. As informações contidas em cada um são enviadas e filtradas pelo National Weather Center (NWC) e posteriormente publicadas no Storm Data pelo National Climate Data Center (NCDC).

Na Europa, através do Tornado and Storm Research Organization (TORRO), a formação de banco de dados de eventos atmosféricos extremos conta com o auxílio de questionários a serem preenchidos por pessoas que presenciaram esses fenômenos. Estão disponíveis dois tipos de questionários: um referente a diferentes tipos de ventos giratórios (whirlwind), como tornado, tromba 
d'água, nuvem funil, redemoinho, etc., e outro referente a tempestades de granizo. Na Alemanha, Áustria e Suíça, existe o sistema de registro de tempestades severas locais denominado de TorDACH. De acordo com Dotzek (2001), esse banco foi iniciado em 1997 com o intuito de registrar todos os eventos de tornados nesses três países. O sistema de registro contém vários tipos de informações sobre os tornados e eventos extremos semelhantes e/ou associados, tais como, microexplosão (downburst), tromba d'água, nuvem funil, granizo, inundações bruscas (flash flood) e redemoinhos (dust and water devil). Um exemplo da estrutura do banco de dados do TorDACH pode ser vista na Figura 4.

\section{Storm Data and Unusual Weather Phenomena}

\begin{tabular}{|c|c|c|c|c|c|c|c|}
\hline Lexption & Dene & $\begin{array}{l}\text { Time } \\
\text { Lecaly } \\
\text { Standard }\end{array}$ & $\begin{array}{l}\text { Puh } \\
\text { Length } \\
\text { (Miks) }\end{array}$ & $\begin{array}{l}\text { Path } \\
\text { Widath } \\
\text { (Yands) }\end{array}$ & $\begin{array}{l}\text { Number of } \\
\text { Persoes of } \\
\text { Killid loiured }\end{array}$ & $\begin{array}{l}\text { Estimated } \\
\text { Danvate } \\
\text { Pugserity Crops }\end{array}$ & $\begin{array}{l}\text { December } 1995 \\
\text { Charaster of Storm }\end{array}$ \\
\hline
\end{tabular}

ALABAMA, North Central

ALZe01>050

Montgomery County

Mengomery

Jacksen County

Francisco

Lee County
Smiths

Sminths

Barbour County

Clio

A blast of Arctic air established several new recoed low temperatures across the northern two-thirds of Alabama. New record lows were set at Huntsville with 12 degroes. Birmingham with 13 degrees. Pinson with 11 degrees, and Montgomery with 19 degroes.

ALABAMA, Southeast

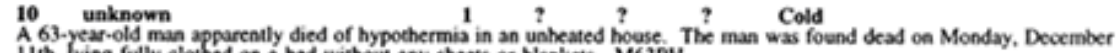
A 63-year-old man apparently died of hypothermia in an unheated house.
IIth, lying fully clothed on a bed without any sheets or blankets. M63PH

10 unknown 1 Cold

County Sheriff's Officers not far from his car which was stock in a creek near the Francisco commenity in extreme nonthwest Jackson County. MS60U

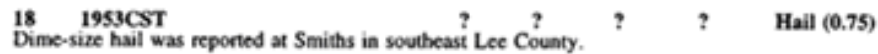

19 0150CST ? ? 45K?

Wind damage was reported in Clio to the roofs of five houses along with damage to several peanut trailers. One house was

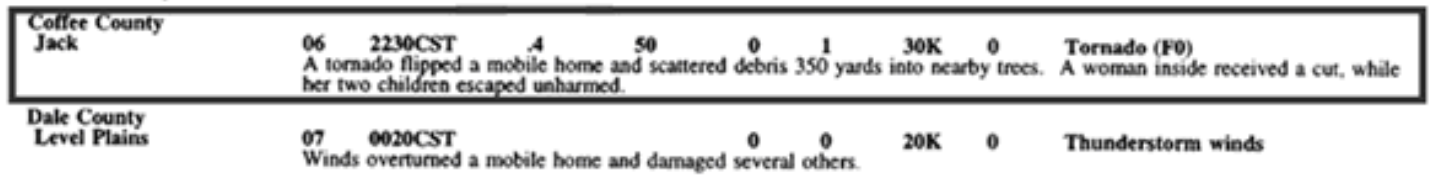

Figura 3. Exemplo da sistematização do banco de dados mensais dos EUA de tempestades e fenômenos de tempo não freqüentes - dezembro de 1995.

Fonte: NCDC (1995).

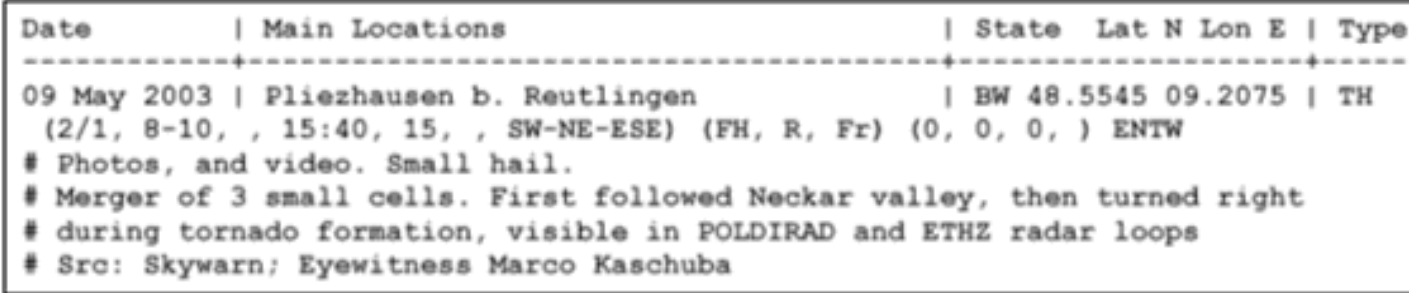

Figura 4. Exemplo de sistematização de informação no banco de dados TorDACH sobre a ocorrência de um tornado.

Fonte: TorDACH (2006). 
Observando a Figura 4, verifica-se que as informações contidas na primeira linha referem-se à: data, localização, posição geográfica e tipo do fenômeno ( $\mathrm{T}=$ tornado, $\mathrm{H}=$ granizo). A linha abaixo informa sobre a intensidade do fenômeno conforme a escala TORRO/Fujita, comprimento da trajetória $(\mathrm{Km})$, largura da trajetória $(\mathrm{m})$, horário, duração total (minutos), principal tempo de impacto (segundos), direção de propagação, morfologia do terreno, tipo de uso da terra, dia da semana, danos em propriedades (Euro), danos ambientais (Euro ou $\mathrm{m}^{3}$ de madeira) e fonte das informações sobre o evento. O símbolo \# indica comentários adicionais relevantes (TorDACH, 2006).

Nota-se que é comum para todos os bancos de dados de tornados o registro da data e horário de ocorrência - fundamental para a caracterização do ambiente atmosférico que desencadeou o fenômeno -, a localização geográfica, as características de superfície e a largura e extensão da trajetória percorrida. As conseqüências do fenômeno também são importantes, uma vez que é extremamente difícil caracterizar a intensidade sem a avaliação de danos. Todavia, essas variáveis de danos requerem uma análise a posteriori no local de ocorrência do tornado e/ou utilização de métodos remotos de análise para extrair informações da trajetória (largura e extensão), tais como fotografias aéreas e imagens de satélite.

Acredita-se que a formação de um banco de dados de tornados para o Brasil é urgente, uma vez que esse fenômeno tem sido cada vez mais freqüente e impactante (OLIVEIRA, 2000; Marcelino et al., 2006; Nascimento e Marcelino, 2005). Além disso, auxiliará diretamente na identificação de um padrão de comportamento atmosférico, que subsidiará os estudos de tempestades severas no território brasileiro. Para tanto, aconselha-se utilizar como modelo os exemplos de banco de dados apresentados, adaptando-os a realidade brasileira.

\section{Análise de tornados utilizando SIG}

A realização de um estudo completo de tornados abrange duas etapas bem distintas: a) origem do fenômeno - que engloba basicamente variáveis geofísicas (meteorológicas e de superfície) e b) danos e prejuízos - utilizando dados físicos (infra-estrutura urbana, edificação, etc.) e socioeconômicos, que estão associados a vulnerabilidade do ambiente exposto (MARCELINO et al., 2004). Na Figura 5 verifica-se uma síntese dessa abordagem geográfica para estudos de episódios de tornados. Vale salientar que um fenômeno atmosférico extremo somente será considerado um desastre natural se atingir um sistema social, causando diferentes tipos de danos e prejuízos socioeconômicos (ALCÂNTARA-AYALA, 2002).

A seguir esta discussão será subdividida em dois sub-itens: o primeiro abordando o uso dessa ferramenta para análise do ambiente atmosférico, abrangendo as etapas de prevenção e mitigação (antes); o segundo item irá considerar as etapas de resposta (durante) e de avaliação de danos (depois). 


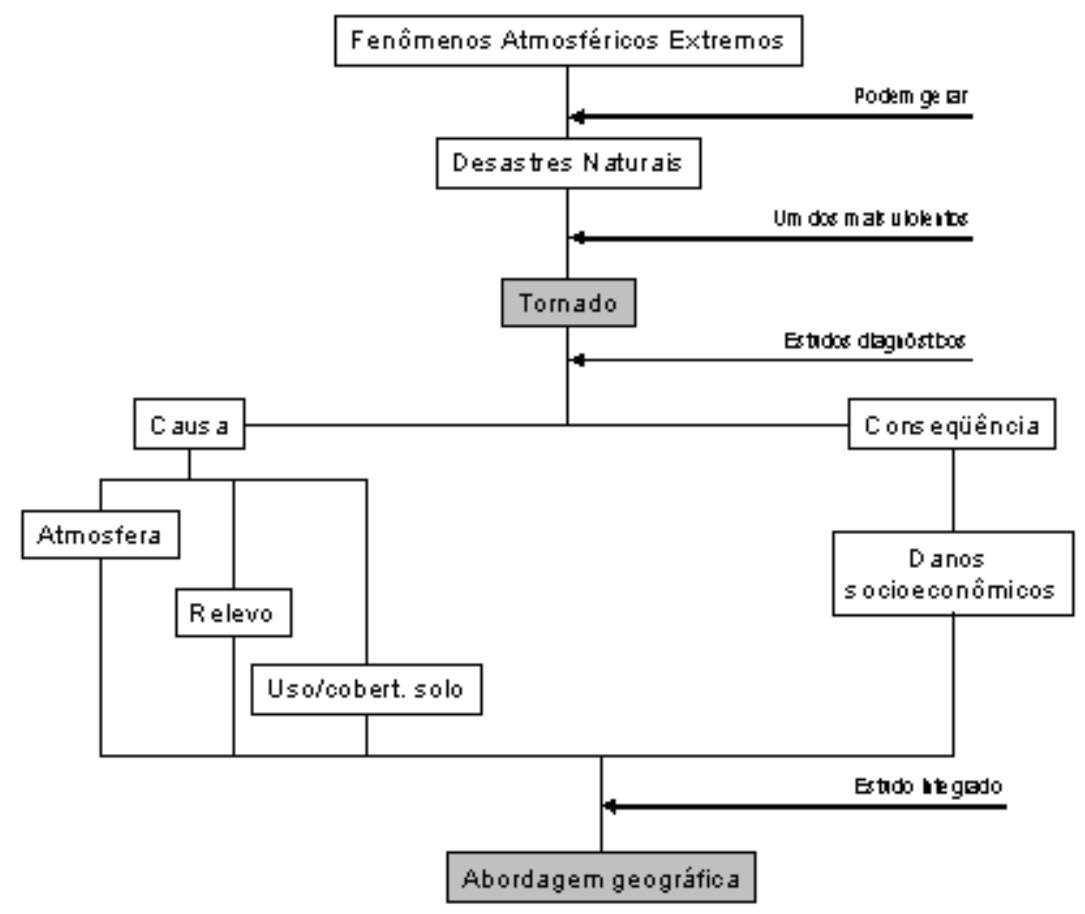

Figura 5. Síntese da abordagem geográfica para análise de episódios de tornados.

Fonte: Adaptado de Marcelino et al. (2004).

\section{Uso de SIG na análise do ambiente atmosférico de tornados}

O SIG tem sido usado para análises meteorológicas e climatológicas em várias partes do mundo. Nos EUA, a adoção dessa ferramenta para a visualização das diferentes variáveis meteorológicas na previsão de tempo severo tem sido amplamente utilizada nas atividades operacionais do National Weather Service. Na Europa foi formada a Acção COST 719, envolvendo 19 países, que tem como objetivo principal analisar as potencialidades do SIG nas áreas de climatologia e meteorologia (PERDIGÃO e MOITA, 2002). A possibilidade de relacionar diferentes variáveis geofísicas em distintas escalas espaciais e temporais produz resultados que são praticamente impossíveis de serem obtidos com aplicativos que permitem somente a análise de um único tipo de variável.

Shipley (2005) comenta que a comunidade de ciências atmosféricas tem desenvolvido visualisadores e aplicativos próprios ao longo dos anos, como o GRADS, FERRET, NCAR graphics. Isso se deve à necessidade de cada disciplina científica criar suas próprias ferramentas focando questões específicas de análise. Entretanto, isso resulta num fator limitante para o cruzamento de dados e informações que, conseqüentemente, irá gerar resultados parciais e segmentados.

Uma das dificuldades encontradas na utilização dessas ferramentas é 
a padronização do formato de extensão dos dados que possibilite a interação no SIG (SHIPLEY, 2005). Alguns institutos nos EUA já vêm trabalhando para a formação de banco de dados atmosféricos em ambiente SIG, denominados por Shipley et al. (2000) como "GIS Ready" databases. O dado meteorológico passa a ser visualizado como ponto, linha, polígono, raster, etc.

A utilização de imagens ópticas orbitais com imagens de radar tem igualmente auxiliado nas análises de tempo severo (SHIPLEY, 2006). Além disso, a identificação de locais adequados para a instalação de radares meteorológicos também tem contado com o auxílio do SIG. Na Figura 6, verifica-se um mosaico de projeções de cinco radares nos EUA, sobrepostos a topografia do terreno (MDT), que visa estimar a cobertura atual de radares e propor uma rede que cubra toda a extensão da região em foco.

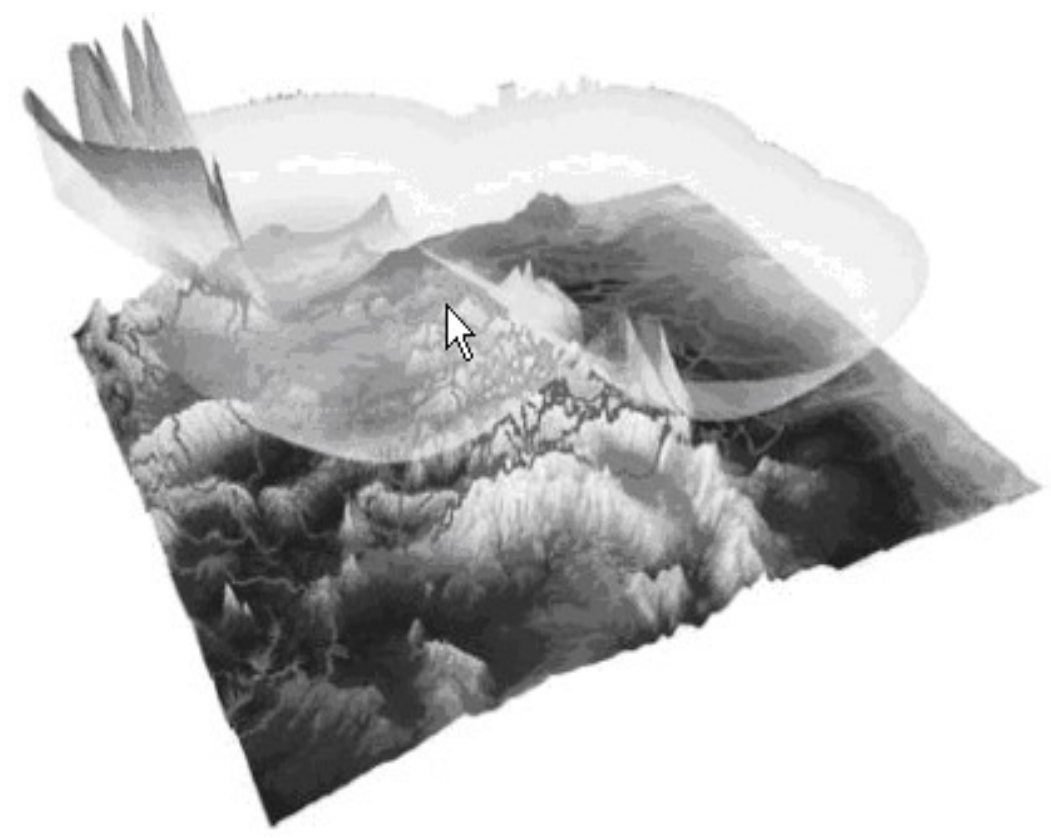

Figura 6. Mosaico da cobertura de abrangência de cinco radares sobre a região central-norte do Colorado (EUA).

Fonte: Shipley et al. (2006).

Dados de modelos de previsão de tempo também estão sendo utilizados em ambiente SIG para serem inter-relacionados com outras variáveis. Na Figura 7, verifica-se o cruzamento da componente zonal (leste-oeste) do vento geostrófico em $850 \mathrm{hPa}$ com a topografia (MDT). O que se encontra em 3D é a topografia que fica acima de $850 \mathrm{hPa}$, e o que não está em 3D, é a que se encontra na figura 7.

A análise espacial é uma das ferramentas largamente utilizada em ambiente SIG e possui um elevado número de recursos de interpolação dos dados atmosféricos. Valeriano e Picini (2000) usaram SIG para gerar a espacialização de modelos de temperatura para o estado de São Paulo, calculados através 
da latitude e altitude. Valeriano et al. (2002) estudaram diferentes métodos de interpolação de dados de precipitação pluviométrica em SIG para esse estado, considerando o efeito de anisotropia e buscando a geração de mapas de confiabilidade conhecida. Nunes (1997) também utilizou essa ferramenta para gerar mapas de precipitação e parâmetros estatísticos derivados para o estado de São Paulo com vistas a detectar tendências recentes na distribuição anual e sazonal desse elemento.

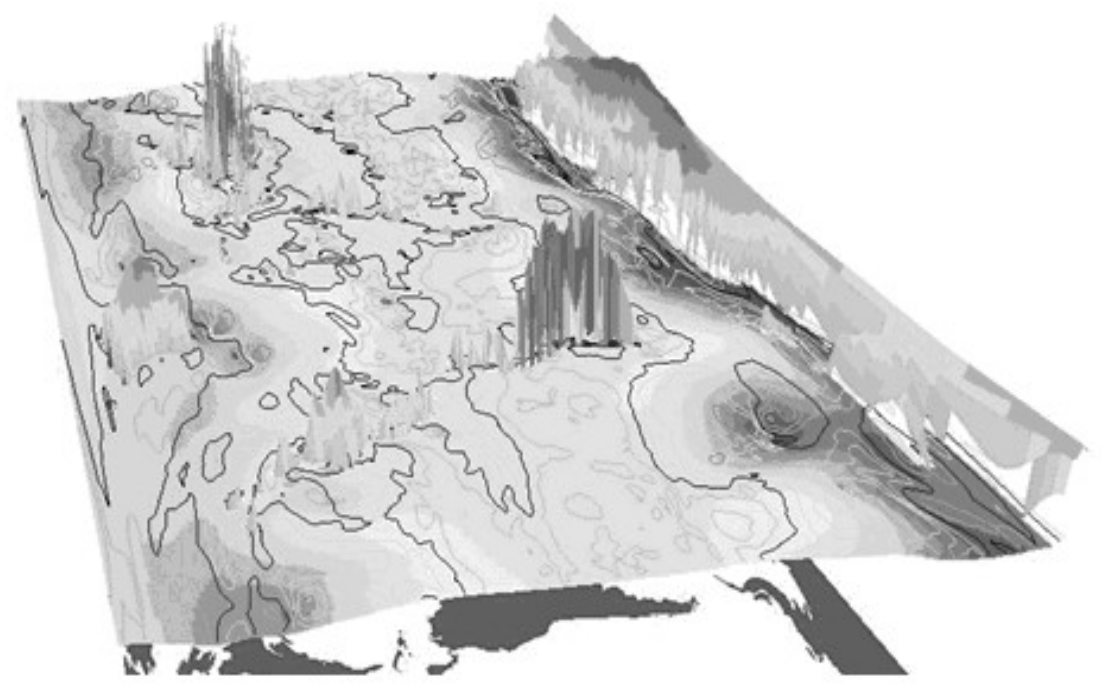

Figura 7. Componente leste-oeste do vento geostrófico em $850 \mathrm{hPa}$ do modelo global MRF associado a topografia acima desse nível em $3 D$.

Fonte: Shipley et al. (2000).

Um dos grupos de trabalho da Acção COST 719 é a de experimentos de interpolação de dados meteorológicos e climatológicos. Esse grupo tem como objetivo estabelecer um inventário de funcionalidades de interpolação já disponíveis em pacotes SIG, analisando as potencialidades e limitações desses interpoladores, comparando-os com outros algoritmos de espacialização, e propondo recomendações para o desenvolvimento do setor (PERDIGÃO e MOITA, 2002).

Em um ambiente SIG também se pode combinar boletins de tempo, entrando no sistema como polígonos, com imagens de satélites, radar, rede de observações de superfície, entre outros. Essa combinação pode fornecer uma visualização da natureza observada e distingui-la da natureza modelada, fundamental para a precisão dos resultados (SHIPLEY, 2006).

Dessa forma, constata-se que a crescente divulgação e utilização do SIG para as análises atmosféricas permitirão que se encontrem novas respostas, interações e formas de trabalho para essa área do conhecimento.

\section{Uso de SIG na análise dos danos de tornados}


Uma das primeiras medidas pós-evento de tornados é a delimitação da trajetória percorrida, com a identificação dos tipos de danos. Nessa fase o Global Positioning System (GPS) torna-se imprescindível, visto que a coleta das coordenadas geográficas em campo é associada com a análise da intensidade do fenômeno em cada ponto da trajetória. Com esses dados em mãos, pode-se facilmente inseri-los em um ambiente SIG, obtendo um mapa de perigo (hazard map), que é a base necessária para a produção de mapas adicionais, como mapas de suscetibilidade, vulnerabilidade e risco.

As informações dos danos coletadas em campo podem ser conjugadas com imagens de satélites ou fotografias aéreas, tornando-se passíveis de serem correlacionadas com outros parâmetros associados ao fenômeno, que se julguem necessários. Por exemplo, Dyer $(1988 ; 1994)$ utilizou imagens do sensor MSS do satélite Landsat e fotografias aéreas para detectar rastros de tornados no sudoeste do Brasil, Paraguai e norte da Argentina. O autor verificou que houve maior dificuldade na identificação dos rastros nos aglomerados urbanos, devido a falta de detalhamento que a resolução espacial das imagens ofereceram.

Alguns pacotes de SIG possuem vários aplicativos para o processamento digital de imagens, permitindo extrair mais informações da trajetória, das condições da superfície do terreno (uso da terra, cobertura vegetal, relevo, etc). Lillesand et al. (2002) realizaram diferentes processamentos digitais de imagens para identificar o rastro de um tornado F3 que ocorreu em junho de 2001 no Estado de Wisconsin (EUA). Para gerar o realce da trajetória e identificar as mudanças produzidas no solo pela passagem do tornado foi empregada a técnica de Análise de Componentes Principais (PCA) (Figura 8). Yuan et al. (2002) também testaram três técnicas distintas de processamento digital de imagens para identificação dos rastros dos tornados de maio de 1999 em Oklahoma (EUA). A melhor técnica para determinar os rastros de tornados menos intensos ( $F 1$ e F2) foi 0 Índice de Vegetação de Diferença Normalizada (NDVI). As demais técnicas realçaram com mais qualidade os tornados de média e forte intensidade.

A Federal Emergency Management Agency (FEMA), órgão oficial dos EUA para atuar na prevenção e resposta aos desastres naturais, vem utilizando SIG desde 1992, quando a costa americana foi atingida pelo Furacão Andrew. Através dos modelos elaborados em SIG, a FEMA toma decisões sobre as possíveis áreas de impacto e suas prováveis conseqüências, antes mesmo da ocorrência do fenômeno. Basicamente, no SIG são correlacionados mapas da trajetória do fenômeno (furacão, tempestades severas, etc.) com mapas da topografia, uso da terra, ressaca (storm surge) e velocidade dos ventos. Além disso, no gerenciamento de risco, são identificadas insfraestruturas que podem ser usadas na fase de resposta, quando são prestados auxílios às áreas afetadas, como: hospitais, postos de saúde, ginásios, escolas, entre outros. Após a ocorrência do desastre, essa agência também utiliza o SIG para mapear os danos, utilizando dados de sensoriamento remoto e/ou informações coletadas em campo (FEMA, 2005a; b). Assim, verifica-se que a utilização dessa ferramenta para o gerenciamento de situações adversas possui um papel amplo nas diferentes etapas do processo, contribuindo para a mitigação dos danos e prejuízos gerados por esses fenômenos.

Fairweather (2005) inseriu em ambiente SIG os rastros dos tornados ocorridos em maio de 1999 em Oklahoma (EUA) com algumas variáveis sociais, 
identificando algumas áreas prioritárias para a resposta pós-desastre. Na Figura 9, verifica-se o cruzamento das áreas prioritárias para atendimento pós-desastre, como também os locais que possuem melhor infra-estrutura para suporte no momento da resposta. Esse cruzamento resultou numa valiosa informação que agiliza $o$ atendimento aos vitimados nas localidades que foram mais atingidas.
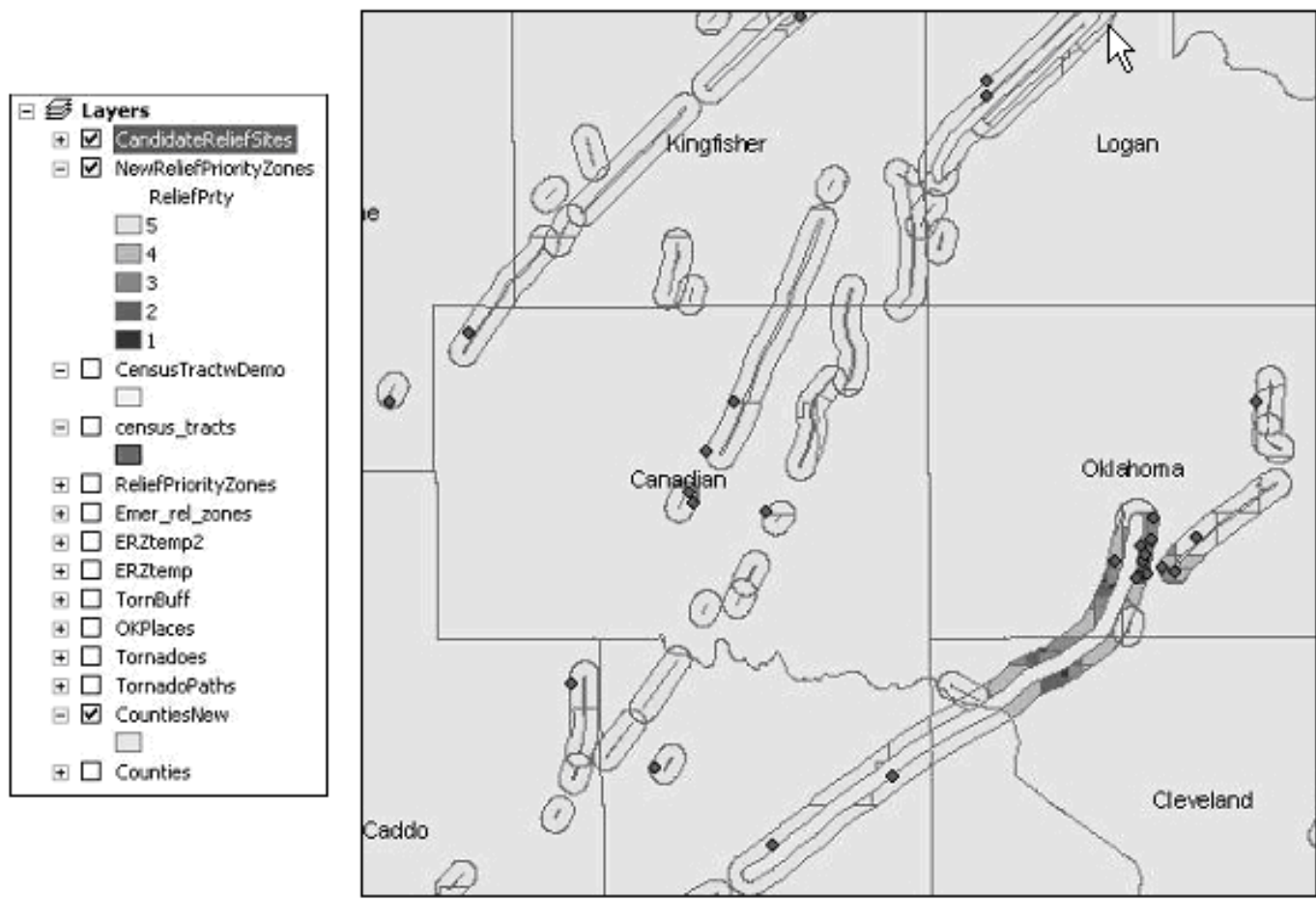

Figura 9. Cruzamento dos rastros dos tornados identificando as áreas prioritárias para atendimento.

Fonte: Fairweather (2005).

Ainda tratando-se da avaliação dos danos de tornados, alguns trabaIhos têm apresentado modelagens da trajetória do fenômeno associado à estrutura urbana das cidades. Isso vem auxiliando as análises de risco, com base em eventos já ocorridos, simulando-os para locais com alta probabilidade de registro. Como exemplo, a Figura 10 mostra resultados de simulações realizadas por NCTCG e NWS (2000), utilizando os dados dos tornados que ocorreram em maio de 1999 em Oklahoma (EUA), para as cidades de Terrant e Dallas, localizadas na região norte do Texas (EUA). Estas simulações permitem estimar os impactos que seriam gerados nessas cidades por um tornado F5, como foi o ocorrido em Oklahoma.

Na Figura 11 foi feito um "zoom" de um trecho da trajetória do tornado em Dallas, em que foram identificadas a quantidade de casas, apartamentos e propriedades comerciais que poderiam ser afetadas, com o respectivo prejuízo na última coluna. 

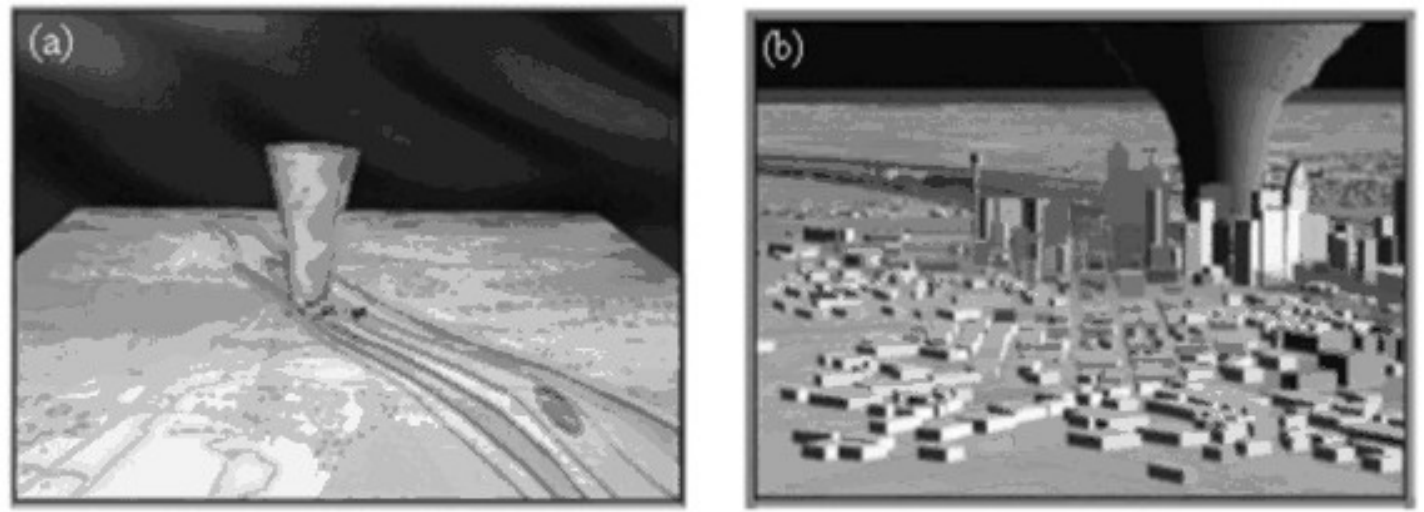

Figura 10. Simulação em SIG da trajetória do tornado mais intenso que ocorreu em maio de 1999 em Oklahoma (EUA): (a) sobre o centro da cidade de Terrant - Texas; (b) no centro da cidade de Dallas - Texas.

Fonte: NCTCG e NWS (2000).

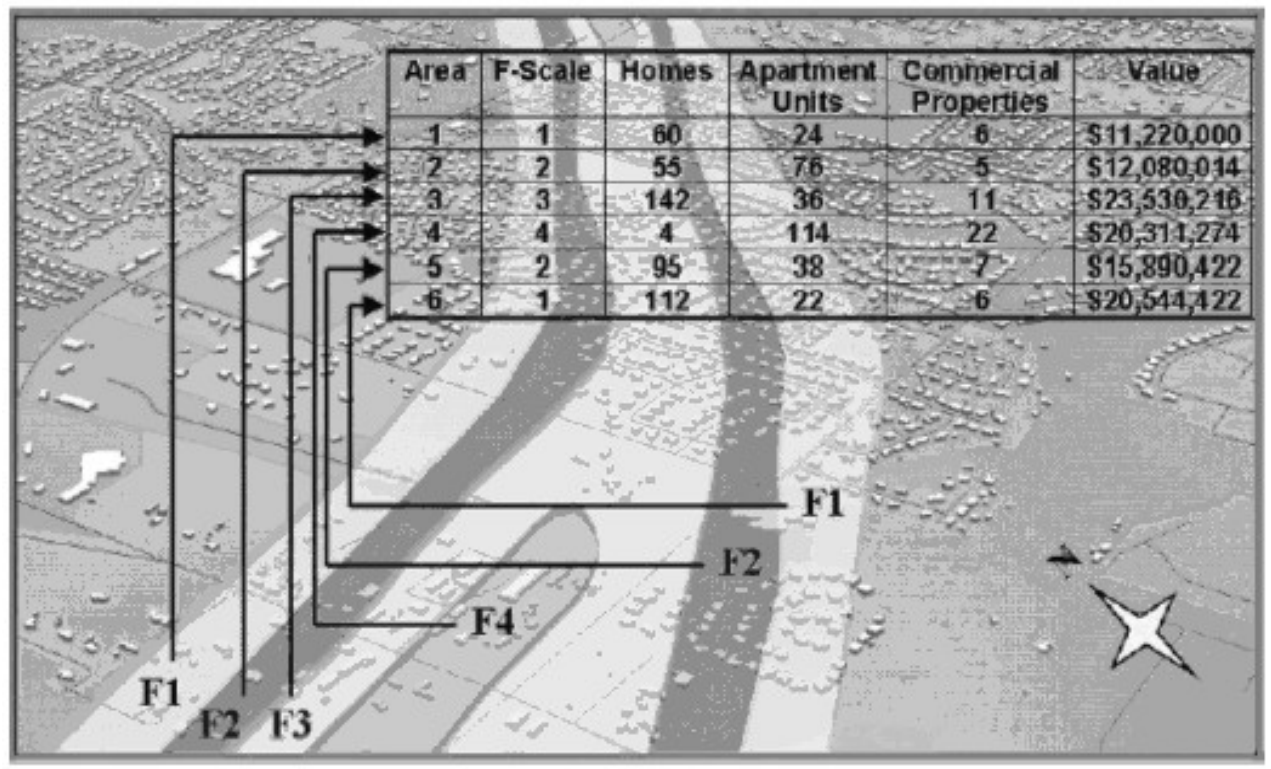

Figura 11. Estimativa dos tipos de danos e prejuízos baseados na simulação do tornado mais intenso ocorrido em maio de 1999 em Oklahoma sobre a cidade de Dallas - Texas (EUA).

Fonte: NCTCG e NWS (2000).

Apesar dessas simulações enquadrarem-se na etapa "antes", auxiliando diretamente na prevenção e mitigação, destaca-se que elas necessitam de informações obtidas pós-evento. Dados sobre os tipos de danos ocasionados, diagnósticos atmosféricos e da superfície terrestre são essenciais para possibilitar a realização desse tipo de simulação. Desta forma, verifica-se que todas as eta- 
pas que envolvem o processo de redução da vulnerabilidade estão correlacionadas e são dependentes entre si. Assim, trabalhar com as diferentes etapas que envolvem um desastre natural utilizando SIG resultará em comunidades mais preparadas para enfrentar fenômenos tão destrutivos como os tornados.

\section{Considerações finais}

O SIG tem mostrado ser uma excelente ferramenta para a análise de desastres naturais, principalmente no gerenciamento de risco e vulnerabilidade. Já nas análises atmosféricas, o SIG tem sido amplamente utilizado nos EUA para análise e modelagem de eventos de tornados, enquanto que no Brasil as aplicações ainda são incipientes e pontuais. Acredita-se que o maior problema é a sistematização de um banco de dados contendo variáveis básicas que possam ser utilizadas nos prognósticos e na definição de medidas mitigadoras e preventivas.

Por se tratar de uma ferramenta de uso multidisciplinar, diferentes variáveis geofísicas e socioeconômicas podem ser inseridas num SIG resultando em análises espaciais, modelagens, mapeamentos, entre outras. No caso dos tornados, sugere-se a adoção de algumas variáveis para a análise do fenômeno, tais como: data, hora, local de ocorrência, intensidade dos danos e largura e extensão da trajetória. Outros atributos que descrevem a configuração da superfície de ocorrência e relatos de pessoas que presenciaram o fenômeno, também são fundamentais nas análises mais detalhadas. Iniciativas semelhantes às adotadas em outros países, como o treinamento de observadores e a utilização de questionários, são métodos eficientes que podem incrementar significativamente o banco de dados.

Apesar das atuais dificuldades, acredita-se ser viável o uso do SIG para a análise desse fenômeno no Brasil, em função do maior interesse da comunidade científica e das agências de fomento por essa temática, o que poderá resultar na formação de uma base de dados confiáveis para o país. Além disso, apesar do presente artigo não ter abordado com maior detalhamento os prognósticos climáticos associados as mudanças climáticas globais, deve-se também levar em consideração suas previsões para o território brasileiro. Visto que uma das principais conseqüências será o aumento de tempestades severas para a região sul e sudeste do Brasil.

Assim, discutir e realizar iniciativas que envolvam a prevenção e a mitigação de fenômenos atmosféricos extremos, como os tornados, deve tornarse cada vez mais freqüentes, visando a preparação e capacitação das comunidades e instituições envolvidas nas diferentes etapas que envolvem o processo de redução da exposição e vulnerabilidade dos sistemas sociais.

\section{Referências bibliográficas}

ALCÁNTARA-AYALA, I. Geomorphology, natural hazards, vulnerability and prevention natural disaster in development countries. Geomorphology, v. 47, n. 2-4, p. 107 124, 2002.

BURROUGH, P. A.; McDONNELL, R. A. Principles of Geographical Information Systems. Nova York: Oxford University Press, 1998. 333 p. 
COPPOCK, J. T. GIS and natural hazards: an overview form a GIS perspective. In: CARRARA, A.; GUZZETTI, F. (Ed.) Geographical information systems in assessing natural hazards. Dordrecht: Kluwer Academic Publishers, 1995. 2, p. $21-34$.

DOSWELL III, C. A.; BOSART, L. F. Extratropical synoptic-sacle processes and severe convection In: Doswell III, C. A. Severe Convective Storms. Boston: American Meteorological Society. Meteorological Monographs, v. 28, n. 50, 2001. p. 27-69.

DOSWELL, C. A.; MOLLER, A. R.; BROOKS, H. E. Storm spoting and public awareness since the first tornado forecasts of 1948. Weather and Forecasting, v. 14, n. 4, p. $544-557,1999$.

DOTZEK, N. Tornadoes in Germany. Atmospheric Research, v. 56, p. 233 - 251, 2001.

DYER, R. A. A review of tornado activity in Brazil. In: INTERNATIONAL SOCIETY FOR PHOTOGRAMMETRY AND REMOTE SENSING (ISPRS), 7., Rio de Janeiro, 1994. Anais... Rio de Janeiro: INPE, 1994. 203-213.

DYER, R. A. Remote Sensing Identification tornadoes tracks in Argentina, Brazil and Paraguay. Photogrammetric Engineering Remonte Sensing, v. LIV, n. 10, p. 1429 - 1435, 1988.

FAIRWEATHER, A. M. Locating tornado relief sites. 2005. Disponível em: $<$ www.personal.psu.edu/users/a/m/amf289/geog483/project3.htm>. Acesso em: dezembro 2005.

FEMA - Federal Emergency Management Agency. GIS developments at FEMA. 2004. Disponível em: <www.gismap.fema.gov/gis02.shtm>. Acesso em: dezembro de 2005a.

FEMA - Federal Emergency Management Agency. How FEMA uses GIS in disaster response. 2004. Disponível em: <www.gismap.fema.gov/gis04.shtm>. Acesso em: dezembro de 2005b.

GLICKMAN, T. S. Glossary of Meteorology. 2nd Ed. Boston: American Meteorological Society, 2000. 855 p.

LILLESAND, T.; PODGER, N.; CHIPMAN, J.; GOLDMANN, R.; LEWELLING, K.; OLSEN, T. Assessing Tornado Damage via Analysis of Multi-temporal Landsat 7 ETM+ Data. In: ANNUAL CONFERENCE OF THE AMERICAN SOCIETY FOR PHOTOGRAMMETRY AND REMOTE SENSING (ASPRS), 22, Washington, 2002. Proceedings... Washington: ASPRS, 2002.

MARCELINO, I. P. V. O.; FERREIRA, N. J.; ANDRÉ, I. N. Análise geográfica do tornado ocorrido no município de Joinville-SC em 31/01/1999. In: SIMPÓSIO BRASILEIRO DE DESASTRES NATURAIS, 1., Florianópolis, 2004. Anais... Florianópolis: GEDN/UFSC, 2004. [CD-ROM].

MARCELINO, I. P. V. O.; HERRMANN, M. L. P; FERREIRA, N. J. The occurrence of tornadoes in Santa Catarina State, Brazil. Australian Meteorological Magazine, 2006. (No prelo).

NASCIMENTO, E. L. Previsão de tempestades severas utilizando-se parâmetros convectivos e modelos de mesoescala: uma estratégia operacional adotável no Brasil? Revista Brasileira de Meteorologia, 20, p. 121-140, 2005.

NASCIMENTO, E. L.; Doswell, C. A. The need for an improved documentation of severe thunderstorms and tornadoes in South America. In: SYMPOSIUM ON THE CHALleNGeS OF SeVERE CONVECTIVE STORMS, Atlanta, 2006. Proceedings... Atlanta: American Meteorological Society, 2006. P18.

NASCIMENTO, E. L.; MARCELINO, I. P. V. O. Análise preliminar dos tornados de 3 de janeiro de 2005 em Criciúma/SC. Boletim da Sociedade Brasileira de Meteorologia, v. 28-29, n. 1, p. 33 - 44, 2005.

NCDC - National Climate Data Center. Storm Data. v. 37, n. 12, 1995. Disponível em: 
<http://www5.ncdc.noaa.gov/pubs/ publications.html>. Acesso em: fevereiro 2006.

NCTCG - North Central Texas Council of Governments; NWS - National Weather Service Fort Worth. Tornado Damage Risk Assessment: Dallas-Fort Worth Metroplex. Arlington/Fort Worth: NWS/Emergency Management Community, 2000. $21 \mathrm{p}$.

NECHET, D. Ocorrência de tornados no Brasil. Boletim da Sociedade Brasileira de Meteorologia, v. 26, n. 2, p. 29 - 39, 2002.

NUNES, L. H. Distribuição espaço temporal da pluviosidade no estado de São Paulo: tendências, variabilidade, processos intervenientes. 1997. 192 f. Tese (Doutorado em Engenharia de Transportes) - POLI, USP, São Paulo, 1997.

OLIVEIRA, I. P. V. Distribuição espaço-temporal e análise de tornados em Santa Catarina no período de 1976 a 2000. In: SIMPÓSIO BRASILEIRO DE CLIMATOlOGIA GEOGRÁFICA, 4., Rio de Janeiro, 2000. Anais... Rio de Janeiro: CLIMAGEO/UFRJ/CREA, 2000. 11 p. (CD Rom).

PERDIGÃO, A.; MOITA, S. A Acção COST 719: a utilização de SIG em climatologia e meteorologia. In: ENCONTRO DE UTILIZADORES DE INFORMAÇÃO GEOGRÁFICA, 7., Oeiras, 2002. Anais... Oeiras: USIG, 2002.

SAMUEL, J.; MCMILLAN, M. E.; CONNELLY, J. B.; SIMS, W. J. GIS applications in the development of natural hazards mitigation risk assessment: an example using tornado events in Arkansas. In: GSA ANNUAL MEETING AND EXPOSITION, Denver, 2004. Abstracts... Denver: Geological Society of American. v. 36, n. 5, 2004, p. 140.

SHIPLEY, S. T. Classroom Exercises in GIS Meteorology. In: SYMPOSIUM ON EDUCATION, 15., Atlanta, 2006. Proceedings... Atlanta: American Meteorological Society, 2006. P1.26.

SHIPLEY, S. T. GIS Applications in Meteorology, or Adventures in a Parallel Universe. Bulletin of the American Meteorological Society, v. 86, n. 2, p. $171-173$, 2005.

SHIPLEY, S. T.; Graffman, I. A.; Ingram, J. K. GIS Applications in Climate and Meteorology. In: ESRI 2000 USERS CONFERENCE, San Diego, 2000. Proceedings... San Diego: ESRI, 2000.

SHIPLEY, S. T.; GRAFFMAN, R. E. S.; FACUNDO, J. GIS Tools for Radar Siting and Analysis. In: INTERNATIONAL CONFERENCE ON INTERACTIVE INFORMATION PROCESSING SYSTEMS FOR METEOROLOGY, OCEANOGRAPHY AND HYDROLOGY, 22., Atlanta, 2006. Proceedings... Atlanta: American Meteorological Society, 2006. 11.3.

TorDACH. TorDACH Germany: usage and format of data. Disponível em: <http://www.tordach.org/de/raw/usage.htm>. Acesso em: maio 2006.

UNDP - United Nations Development Programme. Reducing disaster risk: a challenge for development. New York: UNDP, 2004. 129 p.

VALERIANO, M. M.; PICINI, A. G. Uso de Sistema de Informação Geográfica para geração de mapas de médias mensais de temperatura do Estado de São Paulo. Revista Brasileira de Agrometeorologia, v. 8, n. 2, p. 255 - 262, 2000.

VALERIANO, M. M.; PICINI, A. G.; MELLO, M. H. A. Estratificação de efeitos anisotrópicos do relevo na geração de mapas pluviométricos por geoprocessamento. Revista Brasileira de Agrometeorologia, v. 10, n. 1, p. 167 - 177, 2002.

YUAN M.; DICKENS-MICOZZI, M.; MAGSIG, M. A. Analysis of Tornado Damage Tracks from the 3 May Tornado Outbreak Using Multispectral Satellite Imagery. Weather and Forecasting. v. 17, n. 3, p. $382-398,2002$. 